\title{
Impending Central Retinal Vein Occlusion
}

\author{
Michelle Steenbakkers OD FAAO \\ Correspondence may be directed to: msteenbakkers@hotmail.com
}

\begin{abstract}
Central retinal vein occlusion (CRVO) may present with varied clinical manifestations, ranging from mild blurred vision and scattered retinal hemorrhages to severe vision loss, optic nerve swelling, pronounced retinal hemorrhages, collateral retinal vessel formation and neovascularization. Impending CRVO, also known as partial CRVO, is a relatively poorly-defined sub-classification of the CRVO condition. Those affected are either asymptomatic or may complain of mild, often transient episodes of blurring of vision and present with venous dilation and tortuosity but only a few widely scattered flame-shaped retinal hemorrhages. As an impending CRVO may be the prodromal phase of an acute CRVO, this diagnosis requires careful monitoring of the patient for progression. The following case outlines the differential diagnosis, sequelae and inter-professional management of an impending central retinal venous occlusion.
\end{abstract}

\section{Résumé}

L'occlusion de la veine rétinienne centrale (OVRC) peut produire divers symptômes cliniques variant de la vision floue légère et des hémorragies rétiniennes diffuses jusqu'à une perte de vision sévère, l'œè̀me du nerf optique, des hémorragies rétiniennes prononcées, l'apparition de vaisseaux rétiniens collatéraux et une néovascularisation. L'OVRC imminente, aussi appelée OVRC partielle ou bénigne, est une sous-catégorie relativement mal définie de l'OVRC. Les personnes atteintes sont asymptomatiques ou peuvent se plaindre d'épisodes bénins, souvent transitoires, de vision floue et présenter une dilatation et une tortuosité des vaisseaux sanguins, mais seulement quelques hémorragies rétiniennes très dispersées et en flammèches. Comme une OVRC imminente peut constituer la phase prodromique d'une OVRC aiguë, le diagnostic oblige à suivre attentivement l'évolution du problème chez le patient. Le cas qui suit décrit le diagnostic différentiel, les séquelles et la prise en charge interprofessionnelle d'une occlusion imminente de la veine rétinienne centrale.

\section{Introduction}

Retinal vein occlusion (RVO) is recognized as the second most common vascular disorder after diabetic retinopathy. ${ }^{1,2}$ There are three distinct types of RVO: branch retinal vein occlusion (BRVO), central retinal vein occlusion (CRVO), and hemiretinal vein occlusion, which is an anatomical variant of CRVO. Central retinal vein occlusion can pose a major threat to vision through the potential sequelae of macular edema, retinal ischemia, retinal neovascularization, secondary vitreous hemorrhage, fibrous tissue formation, and secondary neovascular glaucoma. Although CRVO has been recognized as a disease entity for over 150 years, the classification, pathogenesis and management of the condition have remained controversial. ${ }^{3}$
The classic presentation of an acute CRVO includes pronounced dilation and tortuosity of retinal veins, retinal hemorrhages in all quadrants accompanied by extensive retinal edema, cotton-wool spots and optic disc swelling. ${ }^{4}$ As a CRVO affects the main venous outflow trunk, the entire venous system of the retina can become involved. One must also consider that in CRVO a patient may have complete obstruction of the central retinal vein but have well-developed collateral venous channels, such as in the case presented, and manifest only minimal funduscopic changes of venous occlusion. The diagnosis becomes less obvious if the presentation is of either an impending CRVO, less severe CRVO, or if the patient is late in the course of the disease where the dramatic acute findings have resolved over time. In the cases of either an impending 
CRVO or mild CRVO the presenting signs may consist of only tortuous, dilated vasculature, disc hyperemia and swelling and a few scattered hemorrhages. These similarities make distinguishing a mild CRVO from an impending CRVO difficult; monitoring for change over time is required as these conditions may resolve without sequelae, as in the case presented, or an impending CRVO can progress to an acute CRVO requiring treatment.

\section{Case Report}

A 49-year-old male presented to a primary care optometry clinic with episodes of blurred vision in the left eye, each lasting 12 hours. The most recent episode was the night prior and was improving at the time of assessment. The patient stated he was anemic with a normal erythrocyte sedimentation rate, and that blood glucose and $\mathrm{HbA1C}$ as recently assessed by his physician were at the high end of normal. He was taking no medications. Blood pressure measured in office was $130 / 98 \mathrm{mmHg}$.

Best-corrected acuities were 20/15 in each eye; however, a $4 \times 6$ area of cloudy vision was noted superior-temporal to fixation on the Amsler grid for the left eye. Colour vision testing was normal and there was no relative afferent pupillary defect in either eye. Slit lamp examination of the anterior segment was unremarkable in each eye. Goldmann intraocular pressures were equal in each eye at $17 \mathrm{mmHg}$ at 1:00pm. A Humphrey visual field (30-2 SITA Standard) was unremarkable in each eye. A dilated fundus exam revealed bilateral arteriolar attenuation. In the left eye, moderate swelling of the superior aspect of the optic nerve and venous dilation was noted. A tortuous optociliary shunt vessel was also
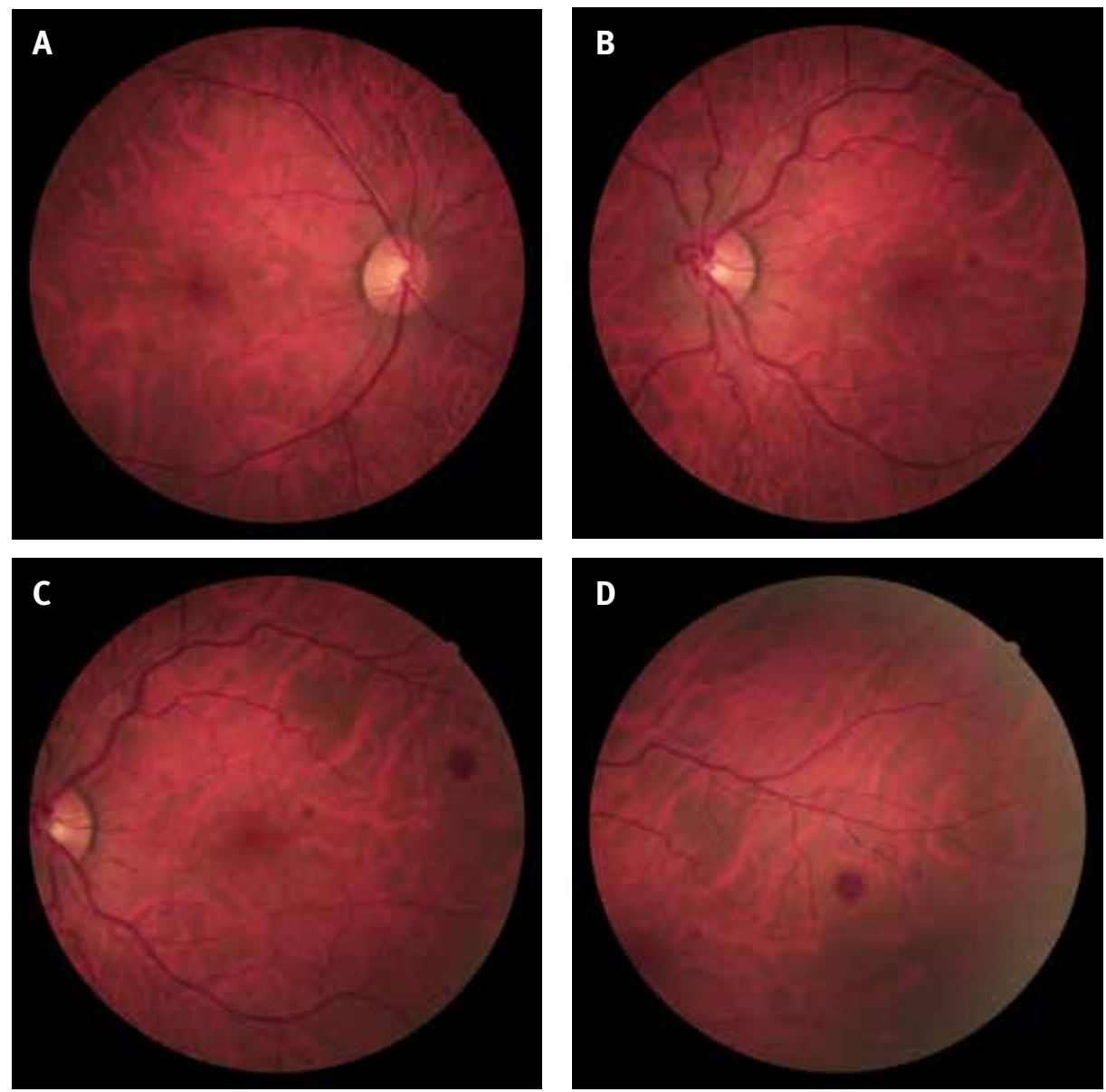

Figure 1. Fundus images at initial presentation of the right $(A)$ and left $(B)$ eyes. Note the decreased arteriolar caliber in the right eye, likely indicative of systemic hypertension. In the left eye there is disc swelling secondary to the impending occlusion, venous dilation and an optociliary shunt vessel. Note the multiple scattered retinal hemorrhages, including a Roth spot, present in the left eye (C,D). 

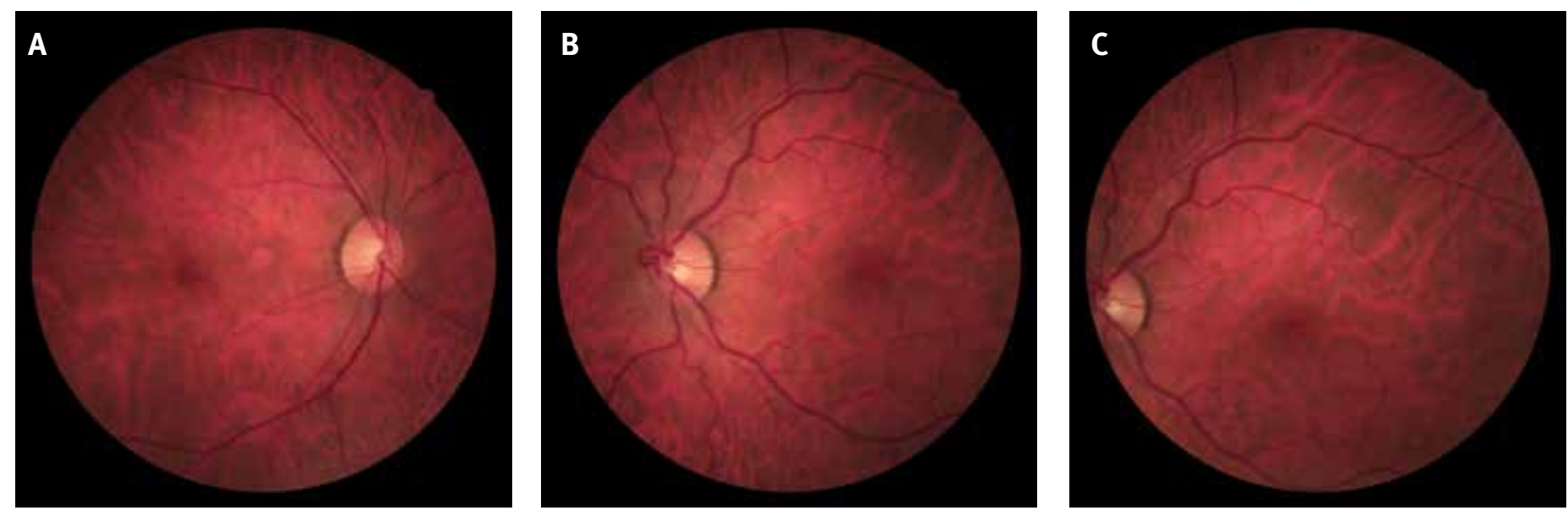

Figure 2. Fundus images 3 weeks after initial presentation in the right $(A)$ and left $(B)$ eyes. Note the significant improvement in the retinal hemorrhages in the left eye and resolution of the Roth spot (C).
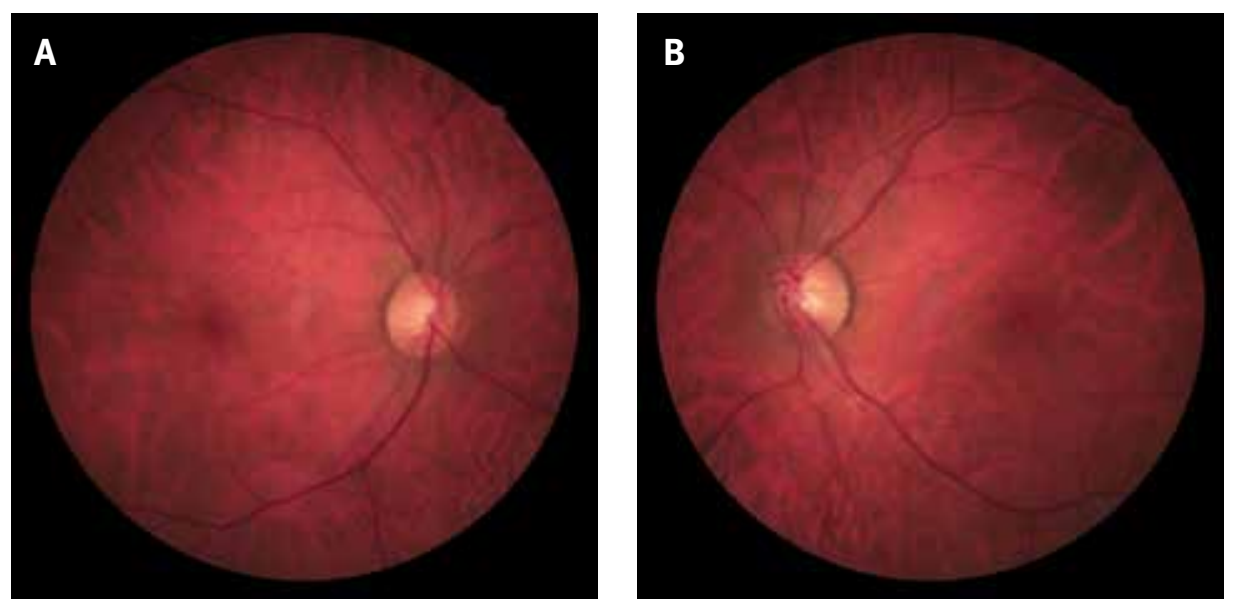

Figure 3. Images of the right (A) and left (B) fundus 8 months after initial presentation. The venous dilation has resolved, and the caliber of the optociliary shunt vessel is reduced.

present at the optic nerve of the left eye. Several dot-blot and retinal nerve fibre layer (RNFL) hemorrhages were noted in the macular area of the left eye, and one larger pale-centered RNFL hemorrhage (Roth spot) was located in the superior temporal arcades (Figure 1). In retrospect, a macular OCT to assess for the presence of macular edema would have been valuable but was not completed.

After the patient's initial presentation, a referral was made to his primary care physician for a full physical examination, including CBC with differentials, blood glucose testing and an assessment for blood hypercoagulation. Given the presence of an optociliary shunt vessel, a CT scan of the head and orbits was performed to rule out any compressive lesion, including optic nerve sheath meningioma. The results of the above testing were unremarkable.

Over the following two months, the patient experienced two additional episodes of blurred vision in the left eye. One episode occurred one week after initial presentation and lasted 24 hours, at which time the visual acuity was $20 / 20$ in the right eye and 20/25 in the left eye. Funduscopic examination was unchanged from the initial presentation one week prior. Three weeks after initial presentation, there was significant resorption of the retinal hemorrhages in the left eye (Figure 2).

The second episode of blurred vision occurred six weeks after initial presentation and lasted 3-4 days. Upon examination, the vision was $20 / 20$ in each eye. Bilateral arteriolar attenuation was noted. In the left eye, there were scattered dot-blot hemorrhages throughout the retina, the tortuous optocilary shunt vessel remained engorged and venous dilation was present. Gonioscopy indicated that there was no neovascularization. During this episode, the patient noted a few neurological symptoms, including numbness in his right leg and foot. Inter-professional collaboration between the optometrist, primary care physician, ophthalmologist and 
neurologist allowed for co-ordinated medical care. A MRI study was not suggestive of multiple sclerosis and the neurological symptoms were attributed to a peripheral nerve injury and not demyelinating disease. A fluorescein angiography was requested but not performed by the ophthalmologist, who instead decided to monitor for progression. A carotid Doppler study was undertaken to rule out carotid artery stenosis as the origin of the asymmetric retinopathy. A 24-hr ambulatory blood pressure to assess for excessive blood pressure variability was also completed. The results of these tests were unremarkable. A further fundus examination at 5 months was more typical of post-venous occlusion, given the venous collaterals noted at the left optic nerve. An evaluation eight months post-initial presentation demonstrated reduction in calibre of the left optociliary vessel and resolution of venous dilation (Figure 3). The patient remained symptom-free at his most recent exam, 16 months after initial presentation.

\section{Discussion}

The pathogenesis of CRVO is not fully understood, but it is currently thought that the condition is the result of a blockage of venous outflow, most likely at or posterior to the lamina cribrosa. It has been hypothesized that the occlusion forms as the result of the central retinal artery compressing the central retinal vein in the area where there is a common fibrous sleeve. This leads to a predisposition for endothelial damage and turbulent blood flow, as evidenced by fluorescein angiography. ${ }^{5}$ In advanced cases of CRVO, histopathologic studies have demonstrated a thrombus formation within the vessel whereby aggregated platelets mesh with crosslinked fibrin protein. It is unknown why there is an affinity for thrombus formation in the central retinal vein at the location of the lamina cribrosa in CRVO, although turbulent blood flow likely plays an important role. ${ }^{5}$ The variability in thrombus formation may explain the variety and severity of presentations of CRVO. ${ }^{4}$ Initial evaluation of a CRVO should include clinical examination and monitoring combined with fluorescein angiography to distinguish between a nonischemic and the more visually threatening ischemic CRVO. These two forms of CRVO and their respective treatment and prognosis are beyond the scope of this review, and are discussed elsewhere. ${ }^{5-7}$

Impending CRVO, also known as partial CRVO, is a relatively poorly-defined sub-classification of the condition which includes patients of a younger age than those typically presenting with CRVO, who are either asymptomatic or who may complain of mild, often transient episodes of blurring of vision. ${ }^{8}$ Most cases of impending CRVO have a good visual prognosis but some advance to complete CRVO, demonstrating that this condition has the potential for bidirectional progress. ${ }^{9}$ The fundus in impending CRVO usually demonstrates mild venous dilation and tortuosity and a few widely scattered flame-shaped retinal hemorrhages, as in the case presented. Some consider impending CRVO as a risk factor for the development of complete CRVO; however, there has been nothing definitive published regarding the natural history of impending CRVO. ${ }^{8,9}$ Furthermore, the patient presented in this report had developed an optociliary shunt which may have spared him from the more classic signs of CRVO.

As mentioned, impending CRVO may resolve or progress to complete obstruction; therefore treatment is aimed at preventing progression to complete occlusion by correcting any predisposing systemic conditions (most commonly including hypertension, diabetes, and/or hyperviscosity), avoiding dehydration, and lowering intraocular pressure (through topical and/or systemic treatment) to improve ocular perfusion. Some advocate antiplatelet agents, and in some circumstances (for example, in a monocular patient) it may be appropriate to consider other options such as anticoagulants, fibrinolytics or hemodilution. ${ }^{7,10}$ Others have suggested that the condition is self-limiting, making invasive investigation (including fluorescein angiography) and treatments (such as those listed above) with possible systemic complications unnecessary. ${ }^{11}$ Instead, the use of a multi-imaging approach including SD-OCT and fundus auto-fluorescence, can be used to detect the fern-like perivenular whitening changes that are characteristic in impending CRVO, and monitor for pro- or re-gression of this sign over time. ${ }^{12}$

Collateral vessel formation is a well-documented sequelae of CRVO. It has been suggested that $70 \%$ of optociliary shunt vessels are attributed to CRVOs. ${ }^{13}$ The shunt vessels typically develop 3-14 months after the occlusion. It has been reported that the presence of these anastomotic vessels following CRVO is associated with a decreased incidence of anterior segment neovascularization. ${ }^{14}$ Others have demonstrated that the presence of an optociliary vessel in non-ischemic CRVO is associated with shorter duration of macular edema. ${ }^{15}$

Optociliary vessels are collateral vessels of a retinochoroidal origin that can be either congenital or acquired. Congenital optociliary shunt vessels direct blood from the choroid to the central retinal vein. In comparison, acquired optociliary vessels direct flow from central retinal vein to the choroid rerouting blood from an obstructed to an unobstructed vein. When the pressure in the central retinal vein becomes greater than that of the choroidal circulation, blood flow is 
Table 1. Differential Characteristics of Retinal Collateral, Shunt and Neovascularization19

\begin{tabular}{|l|l|l|l|}
\hline Vessel Type: & Collateral & Shunt & Neovascularization \\
\hline Origin & $\begin{array}{l}\text { Acquired vessels of capillary } \\
\text { origin, connecting vein-to-vein } \\
\text { or artery-to-artery in area } \\
\text { adjacent to capillary non- } \\
\text { perfusion or vessel blockage }\end{array}$ & $\begin{array}{l}\text { Congenital or acquired } \\
\text { artery-to-vein } \\
\text { communication when } \\
\text { there is no destruction } \\
\text { of the capillary bed }\end{array}$ & $\begin{array}{l}\text { New, thin-walled, fragile } \\
\text { vessels that may be } \\
\text { accompanied by fibrotic } \\
\text { scaffolding }\end{array}$ \\
\hline Appearance & $\begin{array}{l}\text { Tortuous intraretinal vascular } \\
\text { channels }\end{array}$ & Large vascular channel & $\begin{array}{l}\text { Lacy leashes or tufts upon } \\
\text { retinal surfaces }\end{array}$ \\
\hline $\begin{array}{l}\text { Fluorescein } \\
\text { Angiography }\end{array}$ & $\begin{array}{l}\text { Tortuous channel which rarely } \\
\text { leaks }\end{array}$ & $\begin{array}{l}\text { Large, rapidly filling } \\
\text { channel that rarely } \\
\text { leaks, no capillary bed } \\
\text { in area of shunt }\end{array}$ & $\begin{array}{l}\text { Abnormal, tortuous } \\
\text { appearance which leak }\end{array}$ \\
\hline
\end{tabular}

diverted and the optociliary vessel becomes visible. Other names for optociliary shunt vessels include optociliary veins, optociliary collaterals and perhaps most appropriate but not often used, retinochoroidal venous collaterals. In truth, the term optociliary shunt is a misnomer, as these vessels are retinochoroidal rather than optociliary, and are collateral vessels rather than shunts. For a review of collaterals, shunts and neovascularization, refer to Table 1 . In cases of CRVO, the collateral vessel, if present, can be seen on the surface of the optic disc. If the occluded vessel becomes patent over time as the obstruction resolves, the collateral will recede, as was observed in this patient (see Figure 3). ${ }^{16}$

Optociliary shunt vessels can also be present in optic nerve sheath meningiomas, which are associated with optic atrophy and progressive vision loss. ${ }^{16}$ Imaging via CT or MRI scan of the head and orbits can be performed to rule out this condition, as was completed in this patient. If not congenital, in addition to vascular occlusion and optic nerve sheath meningioma, other identified causes of acquired collateral vessel development include optic disc drusen, chronic papilledema, high myopia and diabetes. ${ }^{13,16-18}$

In summary, impending central retinal vein occlusions may be considered a sub-classification along the spectrum of CRVO. It is important to recognize that this condition may represent the prodromal signs of a CRVO, or may resolve without sequelae. In the presence of other confounding factors, such as an optociliary shunt vessel as seen in this case, it is important to rule out other diagnoses while also assessing for underlying risk factors for CRVO. As there is a strong association of CRVO with an underlying systemic condition (dyslipidemia, diabetes, systemic hypertension, cardiovascular disease), it is important to communicate with the patient's primary care physician. The increased use of scanning laser ophthalmoscopy, SD-OCT, and fundus autofluorescence may allow clinicians to monitor this condition in certain cases to avoid more invasive procedures, such as fluorescein angiography.

\section{References}

1. Klein R, et al. The epidemiology of retinal vein occlusion: the Beaver Dam Eye Study. Trans Am Ophthalmol Soc 2000;98:133-41; discussion 141-33.

2. Rogers $S$, et al. The prevalence of retinal vein occlusion: pooled data from population studies from the United States, Europe, Asia, and Australia. Ophthalmology 2010;117:313-19 e311.

3. Hayreh SS. Classification of central retinal venous occlusion. Ophthalmology 1983;90(5):458-74.

4. Gunther J, Scott IU and Ip, M. Retinal Venous Occlusive Disease. In: Albert, DM, ed. Albert and Jakobiec's principles and practice of ophthalmology: 4-volume set. Philadelphia, PA: Saunders Elsevier, 2008: 1755-73.

5. Ho M, et al. Retinal vein occlusions, from basics to the latest treatments. Retina 2016;36(3):432-48.

6. La Spina, et al. Practical management of retinal vein occlusions. Ophthalmol Ther 2012;1(1):3.

7. Parodi MB and Bandello F. Retinal vein occlusion: classification and treatment. Ophthalmologica. 2009;223(5):298-305.

8. Gass JD. Stereoscopic atlas of macular disease: diagnosis and treatment. 4 th ed. St Louis: Mosby; 1997.

9. Lee DH, Lee SJ and Yoon I. Clinical progress in impending central retinal vein occlusion. Korean J Ophthalmol 2010;24(2):83-8.

10. Hayreh SS. Management of central retinal vein occlusion. Ophthalmologica 2003;217:167-88.

11. Paques $M$ and Gaudric A. Perivenual macular whitening during acute central retinal vein occlusion. Arch Ophthalmol 2003;121:1488-90. 
12. Invernizzi A, et al. Multi-imaging interpretation in impending central retinal vein occlusion. Br J Ophthalmol 2013:97(8):1080, 1087.

13. Masuyama Y, Kodama Y, Matsuura Y, et al. Clinical studies on the occurrence and the pathogenesis of optociliary veins. J Clin NeuroOphthalmol 1990;1:1-8.

14. Fuller JJ, Mason III JO, White MF, et al. Retinochoroidal collateral veins protect against anterior segment neovascularization after central retinal vein occlusion. Arch Ophthalmol 2003;121:332-36.

15. Shukiary A, et al. Shunt vessels and outcomes in patients with CRVO treated with bevacizumab. Retina Today 2009;(March):59-61.
16. Haskes $\mathrm{C}$ and Haskes LP. Acquired optciliary shunts and their clinical occurrences. Clin Eye Vis Care 1995;7:69-77.

17. Anderson SF, Townsend JC, Selvin GJ, Jew RL. Congenital optociliary shunt vessels. J Am Optom Assoc 1991 Feb;62(2):109-15.

18. Lee JJ, Yap EY. Optociliary shunt vessels in diabetes mellitus. Singapore Med J 2004 Apr;45(4):166-9.

19. Henkind P and Wise G. Retinal neovascularization, collaterals and vascular shunts. Brit J Ophthalmol 1974;58:413-22.

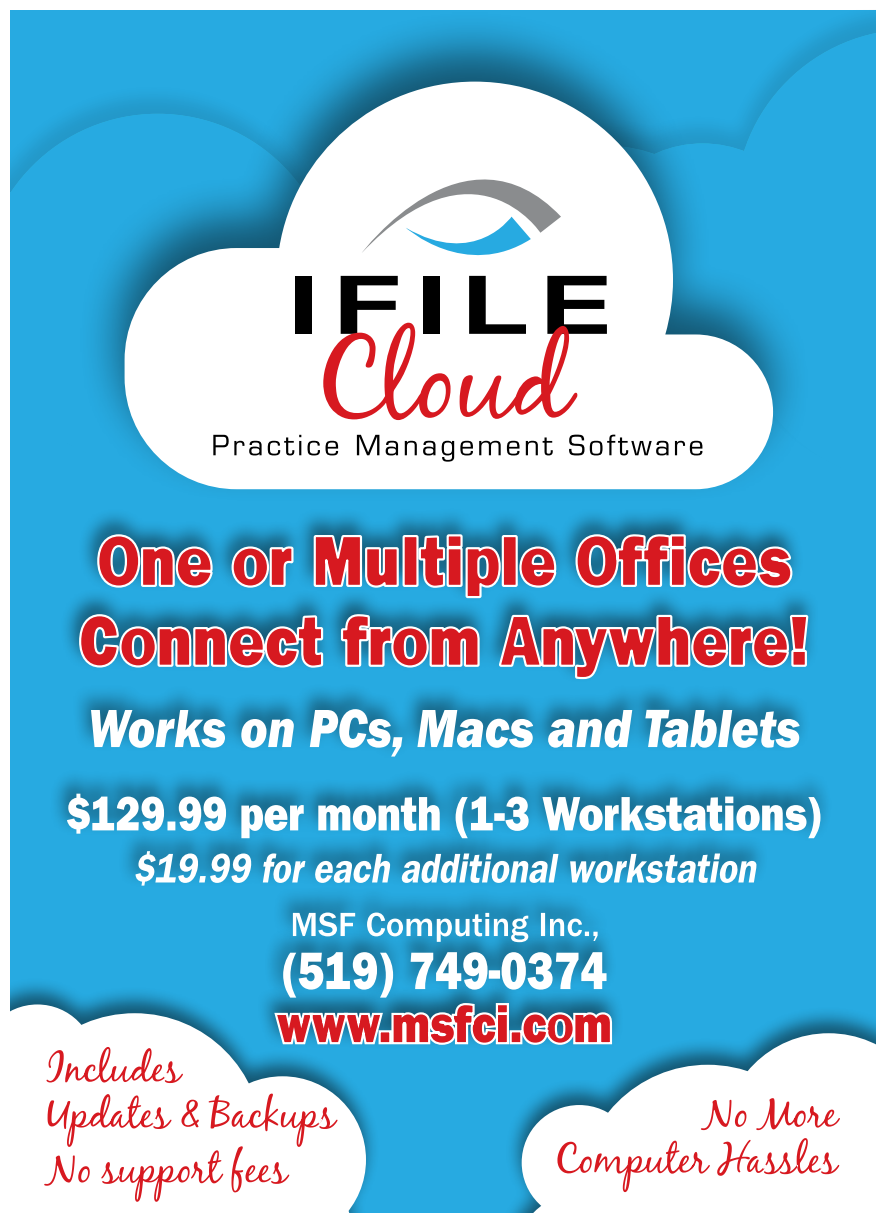

